\title{
Uma análise exploratória de tópicos de pesquisa emergentes em Informática na Educação
}

\author{
An exploratory analysis of emerging research topics in computer science education
}

\author{
Vanessa Araujo Borges \\ Universidade de São Paulo \\ São Carlos, SP - Brasil \\ va.borges@icmc.usp.br
}

\author{
Bruno Magalhães Nogueira \\ Universidade de São Paulo \\ São Carlos, SP - Brasil \\ brunomn@icmc.usp.br
}

\author{
Ellen Francine Barbosa \\ Universidade de São Paulo \\ São Carlos, SP - Brasil \\ francine@icmc.usp.br
}

\begin{abstract}
Resumo Pesquisas que abordam temas sobre Informática na Educação vêm crescendo ao longo dos anos. Em 2012, eventos nacionais relacionados à área, até então não qualificados, receberam conceito Qualis Capes, demonstrando a qualidade e a relevância do que está sendo pesquisado em nível nacional. No entanto, considerando as pesquisas realizadas nos últimos anos, ainda é possível observar que as pesquisas conduzidas no Brasil e exterior possuem diferenças significativas quando analisados os eventos científicos em relação à quantidade de publicações e suas respectivas classificações. Sob essa perspectiva, neste artigo buscou-se investigar o cenário atual da área de Informática na Educação utilizando técnicas de mineração de dados como apoio a extração hierarquia de tópicos. A partir dos dados coletados, foi efetuada uma análise exploratória para identificar os principais assuntos na área de modo a fornecer uma visão geral sobre os tópicos de pesquisa emergentes no contexto educacional.
\end{abstract}

Palavras-Chave: mineração de dados, hierarquia de tópicos, informática na educação

\begin{abstract}
The research in Informatics in Education has attracted a growing interest in the last years. For example, in 2012 Brazilian conferences in this research area received their first qualification score in Qualis Capes, indicating the quality and relevance of the national research in Informatics in Education. However, it is still possible to observe a gap between research conducted in Brazil and in other countries. When comparing national and international conferences, we notice a significant difference in terms of the number of published papers and the Qualis score of the conferences. In this sense, in this paper we investigate the current scenario of the research in Informatics in Education. This investigation has been carried out through the usage of Data Mining techniques to obtain topic hierarchies from the published papers in conferences of the area. An exploratory analysis was carried out to identify the main topics in the area. As a result, we provide an overview of the emerging research topics in the educational contexts.
\end{abstract}

Keywords: data mining, topic hierarchies, computing in education 


\section{Introdução}

Ao longo dos últimos anos, inúmeras pesquisas envolvendo a aplicação de tecnologias computacionais no contexto educacional vêm sendo exploradas com o objetivo de investigar e aprimorar o processo de ensino e aprendizagem. Diversos são os assuntos pesquisados pela comunidade científica visando desenvolver novos métodos, técnicas e ferramentas para atuarem como mecanismos de apoio a esse processo. Tais pesquisas, ao se consolidarem, são divulgadas à comunidade científica, tanto no cenário nacional como internacional, por meio de publicações em anais de conferências e em periódicos.

Em geral, os veículos de publicações científicas são avaliados conforme a importância e o impacto dos trabalhos ali publicados para a comunidade científica. Diversas são as medidas de avaliação de anais e periódicos ao redor do mundo. No Brasil, para classificar a qualidade das produções científicas, a Capes (Coordenação de Aperfeiçoamento de Pessoas de Nível Superior), órgão governamental responsável pela avaliação, acesso e divulgação da produção científica nacional, criou um conjunto de procedimentos, denominado Qualis. Segundo a Capes [3], o Qualis afere a qualidade dos artigos e de outros tipos de produção a partir da análise da qualidade dos veículos de divulgação, ou seja, eventos e periódicos científicos. A classificação de eventos e periódicos é realizada pelas áreas de avaliação e passa por um processo anual de atualização. Esses veículos são enquadrados em estratos indicativos da qualidade - A1 (mais elevado); A2; B1; B2; B3; B4; B5; C (com peso zero).

Considerando, em especial, a área de avaliação de Ciência da Computação que possui linhas de pesquisas com foco em Informática na Educação, existem diversos veículos bem conceituados que buscam, a cada dia, trazer inovações e propor melhorias ao processo educacional. No Brasil, assim como nos demais países, esta área encontra-se em constante crescimento. Na última avaliação de classificação Qualis Capes, divulgada em 2012, alguns eventos e periódicos da área de Informática na Educação até então não qualificados, como WSWED e CINTED, receberam classificações. Além disso, outros eventos tiveram melhora no seu conceito Qualis Capes, como SBIE, WIE e WEI. Isso mostra a crescente importância e a relevância das pesquisas e trabalhos conduzidos na área de Informática na Educação.

No entanto, apesar dos avanços dos últimos anos, ainda é possível observar diferenças significativas entre as pesquisas conduzidas no Brasil e no cenário internacional ao considerar, sobretudo, a quantidade de eventos e periódicos e suas respectivas classificações em ambos os cenários.

Sob essa perspectiva, neste artigo pretende-se fornecer uma visão geral sobre os tópicos de pesquisa emergentes no contexto educacional. Para isso, foram utilizadas técnicas de Mineração de Dados aplicadas em publicações da área de Informática na Educação, divulgadas em eventos nacionais e internacionais, qualificados no Qualis Capes, nos anos de 2011 a 2014. A partir desse conteúdo, foi realizada uma análise exploratória, por meio da geração automática de uma hierarquia de tópicos, dos temas de pesquisa mais recorrentes dentro das comunidades nacional e internacional.

Adicionalmente, é apresentada uma análise que descreve a aderência das pesquisas realizadas no âmbito nacional em relação aos tópicos emergentes e em relação ao que tem sido pesquisado no contexto internacional. Espera-se, com isso, apresentar o cenário atual da área de Informática na Educação considerando seus principais eventos científicos e os assuntos de interesse mais investigados no período de 2011 a 2014.

Pesquisas semelhantes a esta, como por exemplo as realizadas por Romero e Ventura [21], Murray [23], possuem uma visão geral do estado da arte de assuntos relacionados $\mathrm{a}$ áreas específicas e não da área de Informática na Educação como um todo. Além disso, tais pesquisas não possuem análises que demonstram diferenças nos contextos nacional e internacional.

Este artigo está estruturado da seguinte forma: na Seção 2 é descrito o processo de Mineração de Dados, com ênfase na extração de hierarquias de tópicos. Na Seção 3 é descrita a etapa de coleta dos documentos e algumas análises preliminares dos mesmos. Na Seção 4, é detalhado o processo de mineração de extração de hierarquia de tópicos considerando os documentos coletados. Na Seção 5 são descritos os assuntos explorados a partir da hierarquia de tópicos. Por fim, na Seção 6 os resultados obtidos são sumarizados.

\section{Mineração de dados e hierarquia de tópicos}

Uma das linhas de pesquisa que vem apoiando a descoberta de conhecimento é a área de Mineração de Dados. A Mineração de Dados é um processo de análise e descoberta de dados que, sob limitações aceitáveis de eficiência computacional, produz uma determinada enumeração de padrões a respeito dos dados [26]. Esse processo pode ser automático ou semiautomático e os padrões descobertos devem ser novos, representativos, não triviais e levar a alguma vantagem [11].

O processo de Mineração de Dados é composto por cinco etapas principais [22]: (i) Identificação do Problema; (ii) Pré-Processamento; (iii) Extração de Padrões; (iv) Pós- 
Processamento; e (v) Uso do Conhecimento. Essas etapas contêm atividades que buscam garantir desde a integridade dos dados, passando pela correta extração de padrões a partir desses dados, até a validação e uso do conhecimento extraído.

A descoberta de conhecimento em dados de diferentes naturezas apresenta desafios advindos das particularidades desses dados. Com isso, é necessário especializar e adequar o processo de Mineração de Dados, de acordo com a necessidade. Para a exploração de conhecimento a partir de coleções textuais, a especialização do processo de Mineração de Dados é conhecida como Mineração de Textos [17]. Na Mineração de Textos, o grande desafio consiste em obter, para as coleções de documentos, uma estruturação processável por métodos de extração de padrões. Algoritmos utilizados na etapa de Extração de Padrões em processos de Mineração de Dados de qualquer natureza requerem que os dados estejam em um formato estruturado, isto é, sejam definidos os exemplos e um conjunto de atributos representativos que os descrevam.

O principal exemplo de tal estruturação é a matriz atributo-valor, na qual cada linha corresponde a um exemplo e cada coluna a um atributo [22]. Como em Mineração de Textos não se sabe a priori quais são os atributos que descrevem cada documento, é preciso um processamento adicional com essa finalidade. A estruturação para os dados é obtida na etapa de Pré-Processamento, sendo nesta etapa que se encontra a principal diferença entre os processos de Mineração de Textos e Mineração de Dados [2]. Nas demais etapas, as técnicas e algoritmos a serem utilizados são os convencionais.

Uma aplicação interessante da Mineração de Textos refere-se à detecção de tópicos presentes em coleções de documentos, visando organizar essas coleções sob grupos significativos $[7,12,28]$. Nessas aplicações, assume-se que cada grupo de documentos refere-se a um mesmo tema. Para cada grupo, então, seleciona-se um conjunto de termos que descrevem o tema nele retratado. A cada um destes descritores dá-se o nome de tópico.

Dentre os métodos existentes para a obtenção de tópicos em coleções textuais, destaca-se o uso de agrupamento hierárquico dos documentos [1]. A disposição hierárquica dos grupos possibilita detectar tópicos e subtópicos na coleção textual. Em hierarquias de tópicos, grupos que se encontram em níveis mais altos da hierarquia representam tópicos mais gerais, enquanto que grupos em níveis mais baixos representam tópicos mais específicos, ou seja, são subtópicos de tópicos mais gerais. De acordo com Garcia e Porrata [20], as hierarquias de tópicos são a forma mais intuitiva de organização de tópicos, fornecendo visões dos dados em diferentes níveis de abstração. Ainda, segundo Feldman [18], as hierarquias de tópicos representam um papel importante na recuperação da informação, principal- mente quando se deseja efetuar buscas exploratórias.

Hierarquias de tópicos são utilizadas no auxílio à gestão de conhecimento em diversos contextos, como perfis de usuários [24], notícias [7,20,14,15], resultados de motores de busca de páginas web [7,14] e dados biológicos [28]. Em todas essas aplicações, os resultados reportados sobre o uso de hierarquias de tópicos indicam a eficácia dessa estrutura em sumarizar o conhecimento contido em bases textuais.

No contexto de Informática na Educação, não foram encontradas pesquisas que analisam a área como um todo. De modo geral, as pesquisas encontradas abordam assuntos a partir de revisões sistemáticas da literatura ou análise do estado da arte em contextos específicos [6,25,5]. Essas pesquisas são baseadas em questões, a partir das quais é feita uma revisão sistemática da literatura. Neste trabalho, ao contrário de uma revisão sistemática, a metodologia proposta aborda a área de forma mais ampla, partindo de um conjunto de documentos com o objetivo obter os assuntos mais relevantes da área.

Motivados pelos bons resultados alcançados a partir da utilização de hierarquia de tópicos, e considerando a ausência de pesquisas mais abrangentes na área de Informática na Educação, neste trabalho é apresentada uma metodologia para obtenção de uma hierarquia de tópicos por meio de Mineração de Textos. As hierarquias de tópicos são geradas a partir dos artigos científicos publicados em veículos da área, nos contextos nacional e internacional.

Nas próximas seções são descritos os passos realizados durante a coleta e análise dos documentos, a fim de explorar as informações para obter uma melhor compreensão do que está sendo pesquisado e discutido pela comunidade científica.

\section{Análise e coleta de artigos de confe- rências em Informática na Educação}

Um dos principais aspectos para se obter bons resultados em processos de Mineração de Textos diz respeito à qualidade dos documentos utilizados como entrada no processo [2]. Deve-se procurar garantir a representatividade dos documentos utilizados, isto é, que os documentos consigam representar, consistentemente, o conhecimento do domínio a ser explorado.

A fim de possibilitar a extração de conhecimento a partir das produções publicadas, inicialmente foi feita uma seleção dos principais eventos na área de Informática na Educação. Para isso, considerou-se os estratos Qualis Capes divulgados na última avaliação realizada, correspondente ao ano de 2012. A busca por eventos da área foi feita em ordem decrescente de estrato, dos mais bem qualificados àqueles com menor classificação. 
A partir dos eventos selecionados, buscou-se pelos artigos publicados em cada um dos eventos nos anos de 2011 a 2014 considerando aqueles que já divulgaram seus documentos até a data desta análise. Esse intervalo de tempo foi selecionado por considerar que as publicações deste período ainda são atuais, bem como pelo fato do número de documentos ser satisfatório para alcançar uma boa análise. $\mathrm{Na}$ Tabela 1, são listados os eventos selecionados, sua classificação Qualis (estrato) e a quantidade de documentos/artigos coletados para cada um deles.

Considerando os eventos selecionados, é importante observar que:

- O número de eventos nacionais que investigam temas relacionados à área de Informática na Educação com classificação Qualis é de apenas 5. Com isso, foi necessário limitar a quantidade de eventos internacionais, também em 5 , embora existam aproximadamente 60 outros eventos internacionais relacionados à área. Essa limitação foi necessária para garantir que a análise fosse feita considerando os mesmos critérios em ambos os cenários.

- O estrato Qualis dos eventos internacionais são classificados de $\mathrm{A} 2$ a $\mathrm{B} 2$ enquanto que os nacionais estão entre B2 e B5. Isso demonstra que, considerando o conjunto de procedimentos definidos pela Capes, os eventos internacionais, em geral, possuem publicações de maior impacto na comunidade acadêmica.

Tabela 1: Eventos selecionados para análise.

\begin{tabular}{|l|c|c|c|}
\hline \multicolumn{1}{|c|}{ Sigla } & Estrato & Tipo & Qtd. Doc. \\
\hline SBIE $^{1}$ & B2 & N & 387 \\
\hline WIE $^{2}$ & B3 & N & 167 \\
\hline WEI $^{3}$ & B4 & N & 158 \\
\hline CINTED $^{4}$ & B5 & N & 288 \\
\hline WSWED $^{5}$ & B5 & N & 13 \\
\hline SIGCSE $^{6}$ & A2 & I & 520 \\
\hline ITiCSE $^{7}$ & B1 & I & 425 \\
\hline FIE $^{8}$ & B1 & I & 209 \\
\hline ICALT $^{9}$ & B1 & I & 1063 \\
\hline CSEE $^{10}$ & B2 & I & 823 \\
\hline
\end{tabular}

Vale ressaltar que, mesmo em nível internacional, não existem conferências classificadas como A1 na área de Informática na Educação. Conferências como ACM-

\footnotetext{
${ }^{1}$ Simpósio Brasileiro de Informática na Educação

${ }^{2}$ Workshop sobre Informática na Escola

${ }^{3}$ Workshop sobre Educação em Informática

${ }^{4}$ Ciclo de Palestras Novas Tecnologias na Educação

${ }^{5}$ Brazilian Workshop on Semantic Web and Education

${ }^{6}$ Technical Symposium on Computer Science Education

${ }^{7}$ Annual Conference on Integrating Technology into Computer Science Education

${ }^{8}$ Frontiers in Education Conference

${ }^{9}$ International Conference on Advanced Learning Technologies

${ }^{10}$ Conference on Software Engineering Education and Training
}

$\mathrm{SAC}^{11}$, classificada recentemente como A1 (2012), possuem eventos organizados em trilhas que se alteram a cada edição, o que não garante que a área de Informática na Educação seja sempre contemplada. Por esse motivo, tais eventos não foram considerados neste trabalho.

Além disso, se analisada a quantidade de produções publicadas em cada evento, observa-se que o número de publicações nacionais (1013 documentos) é aproximadamente

\section{Quantidade de documentos por evento}
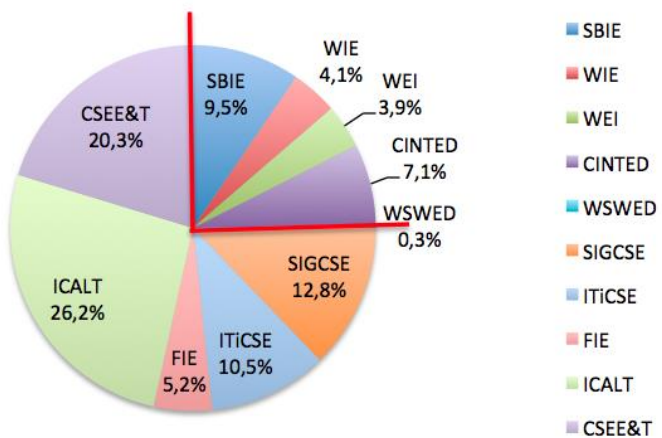

Figura 1: Percentual da quantidade de documentos por evento selecionado.

um terço da quantidade publicada em eventos internacionais (3040 documentos), conforme ilustrado na Figura 1.

\section{Agrupamento dos documentos e ex- tração de tópicos}

Conforme discutido na Seção 3, para a avaliação experimental foi utilizado um total de 4053 documentos publicados em 5 eventos nacionais e 5 eventos internacionais. Estes documentos foram aplicados a uma instanciação do processo de Mineração de Textos com a finalidade de obter uma hierarquia de tópicos a partir dos mesmos.

Os procedimentos relativos à delimitação do problema a ser tratado e à coleta de documentos que atendam a esse problema, também descritos na Seção 3, correspondem às duas primeiras etapas da Mineração de Textos. Passa-se, então, às etapas que visam garantir a adequação e qualidade dos dados e, posteriormente, à extração e validação dos padrões. Desse modo, o restante do procedimento experimental deste trabalho foi dividido em três etapas, de acordo com o processo de Mineração de Textos: (1) préprocessamento dos textos; (2) extração de padrões; e (3) pós-processamento.

\footnotetext{
${ }^{11}$ Symposium On Applied Computing
} 


\subsection{Pré-processamento de textos}

O objetivo do pré-processamento em Mineração de Textos é transformar os documentos em um formato manipulável pelos algoritmos de extração de padrões [22]. A maioria dos algoritmos de extração de padrões atua sobre representações formato atributo-valor.

De acordo com Nogueira et al. [2], uma primeira tarefa a ser realizada na etapa de pré-processamento dos documentos refere-se à padronização dos documentos, na qual deve-se garantir a adequação dos documentos aos algoritmos a serem utilizados. Neste trabalho, todos os documentos considerados são artigos científicos em seu formato publicado, uma vez que foram extraídos dos sites oficiais. Assim sendo, todos os documentos estão em formato Portable Document Format (PDF).

A fim de tornar as informações dos documentos acessíveis e manipuláveis, a primeira atividade realizada na etapa de pré-processamento foi a transformação destes documentos para o formato de texto plano (TXT). A partir dos documentos transformados, foi possível executar os algoritmos de Mineração de Texto de forma automatizada.

Com os documentos em um formato padrão manipulável, foi efetuado um processo de padronização dos termos. O objetivo desta etapa é gerar os atributos que melhor descrevem a base de dados. Em Mineração de Textos, os atributos que representam uma coleção de documentos são também conhecidos como termos e são gerados a partir das palavras contidas em cada documento. Considera-se cada palavra presente na coleção de documentos como candidata a termo. No entanto, nem todas as palavras contidas nos documentos são representativas, isto é, nem todas trazem informações novas e importantes acerca do tema dos documentos da coleção. Dessa forma, deve-se aplicar um processo cuidadoso na geração desses termos, formando um conjunto de termos mais conciso e representativo possível.

Neste trabalho, tal como sugerido em Nogueira et al. [2], optou-se por um procedimento de geração de termos constituído de duas tarefas: remoção de stopwords e radicalização das palavras. Stopwords são palavras que devem ser desconsideradas por não trazerem informação significativa para caracterizar o conteúdo principal dos documentos, como artigos, preposições, pontuações e numerais. Já a radicalização consiste na redução das palavras ao seu radical. Por exemplo, as palavras pesquisador, pesquisa e pesquisou são reduzidas ao mesmo radical pesquis. Este processo é importante para evitar a geração de muitos atributos com grafias diferentes, mas significado semântico semelhante [4]. A radicalização foi feita por meio do algoritmo de Porter [16], o qual possui implementações tanto para a língua portuguesa quanto para a língua inglesa. Tanto a remoção de stopwords quanto a radicalização foram realizadas com o apoio da ferramenta PreText [8].
Mesmo com um criterioso processo de geração de termos, o número de termos gerados é, em geral, muito grande. Para a aplicação de algoritmos de extração de padrão em processos de Mineração de Textos, um grande número de atributos pode tornar o processo computacionalmente inviável, além de prejudicar o desempenho desses algoritmos [2]. Assim, é necessário reduzir o número de atributos presentes na coleção. Optou-se por efetuar uma seleção dos atributos pela sua frequência absoluta, descartando termos muito frequentes ou pouco frequentes por meio do Corte de Luhn [10]. Termos com alta frequência tendem a ocorrer em grande parte dos documentos, não ajudando a distinguir o conteúdo dos documentos. Por outro lado, termos com baixa frequência tendem a apresentar ocorrências isoladas, não ajudando a distinguir grupos de documentos presentes na coleção.

Com o auxílio da ferramenta PreText, termos que apareciam menos de 10 ou mais de 500 vezes ao longo da coleção foram descartados. Assim, foram selecionados 550 termos para a base de dados com documentos de publicações nacionais, enquanto 1493 termos foram selecionados para representar os documentos da base de dados com publicações internacionais.

Uma vez selecionados os termos que representam a coleção de documentos, esta pode ser estruturada na matriz atributo-valor. No preenchimento da matriz, escolheu-se a medida TFIDF (Term Frequency - Inverse Document Frequency) [9] para relacionar termos e documentos. Ao ponderar a frequência de um termo pelo inverso do número de documentos em que este termo ocorre, a TFIDF garante pesos menores a termos que ocorrem em poucos documentos. Dessa forma, a TFIDF consegue mensurar o poder discriminatório de um termo, sendo, por isso, uma medida amplamente utilizada na literatura de Mineração de Textos, e obtendo bons resultados na representação de documentos [4].

\subsection{Extração de padrões}

Com o pré-processamento concluído, tem-se a matriz atributo-valor corretamente formada. Segue-se, então, para a etapa de extração de padrões, na qual são obtidos os padrões a partir dos dados, de acordo com o objetivo do processo de Mineração de Textos [21].

Neste trabalho, o objetivo consiste em extrair hierarquias de documentos, de maneira a organizá-los em grupos representativos. Assim, optou-se por utilizar algoritmos de agrupamento hierárquico de documentos [1], os quais obtêm partições aninhadas da base de dados. Mais especificamente, optou-se por utilizar o algoritmo Bisecting $K$-Means, pela sua simplicidade computacional e pelos bons resultados que apresenta em avaliações experimentais presentes na literatura [27]. Este algoritmo consiste na aplicação aninhada do algoritmo K-Means [13], adotando o 
fator de ramificação $(k)$ como 2. Para a aplicação do algoritmo de agrupamento, utilizou-se a distância de cosseno para calcular distâncias entre documentos, uma vez que essa é a mais indicada para processos de Mineração de Textos, por ser bastante adaptável a contextos esparsos [4].

Para cada execução do algoritmo $K$-Means, 10 inicializações diferentes do algoritmo foram utilizadas, uma vez que o algoritmo tem inicialização aleatória. A cada inicialização, o algoritmo tinha como critério de parada a realização de 50 iterações (valor escolhido empiricamente). Ao final, dentre todos os agrupamentos gerados pelas diferentes inicializações, escolhia-se aquele com melhor valor de silhueta [1]. A medida de silhueta é um índice de critério relativo utilizado para avaliar partições, verificando o quão bem os documentos estão situados dentro de seus grupos. O valor da silhueta é calculado para cada documento da coleção, estando situada no intervalo $[-1,1]$. Valores negativos indicam que os documentos estão erroneamente agrupados, enquanto valores positivos indicam o correto agrupamento do documento. Para avaliar um agrupamento, isto é, uma execução do algoritmo Bisecting $K$-Means, faz-se uma média da medida de silhueta obtida por todos os documentos.

Uma vez obtido o agrupamento final (dendrograma), este pode ser refinado. Em geral, seres humanos têm maior facilidade de lidar com categorias mais gerais (nos níveis mais altos da hierarquia), uma vez que em categorias mais específicas é necessário um grande conhecimento especialista para identificar o conteúdo das categorias [19]. Assim, procurou-se formar hierarquias de menor profundidade e maior largura. Para esse refinamento, foi utilizada a medida de fusão com valor de limiar 0.3 . Um baixo valor de fusão indica grande similaridade entre clusters pai e filho. Nesses casos, ambos os clusters descrevem padrões similares, devendo o cluster pai ser removido e todos os seus filhos serem promovidos ao nível do pai.

A aplicação do algoritmo de agrupamento hierárquico bem como o refinamento da hierarquia obtida foram realizados com o apoio da ferramenta TopHClust ${ }^{1}$. Com essa ferramenta, é possível gerar hierarquias de tópicos de maneira a organizar e analisar o conhecimento implícito em coleções textuais. Os documentos são organizados em tópicos e subtópicos com o objetivo de possibilitar aos usuários explorar dados textuais em diferentes níveis de granularidade.

\subsection{Pós-processamento dos padrões obtidos}

Com o dendrograma gerado e refinado, foi possível visualizar a hierarquia gerada e validá-la, isto é, verificar se ela se encontra de acordo com o domínio de Informática na

\footnotetext{
${ }^{1} \mathrm{http} / / /$ sites.labic.icmc.usp.br/torch/msd2011/tophclust/
}

Educação. Para essa visualização, foi utilizada a ferramenta Torch [19], uma aplicação Java que permite a visualização de hierarquias de tópicos formada a partir de documentos por meio de árvores hiperbólicas e pastas de navegação.

Os tópicos das hierarquias foram formados por descritores dos clusters presentes nessas hierarquias. Para tal, foram escolhidos como descritores, para cada cluster, os 20 termos mais frequentes. Segundo Koller e Sahami [6], o uso de 10 a 25 palavras-chave é suficiente para representar o conteúdo de um documento, tanto em tarefas de agrupamento como de classificação.

Com isso, encerra-se a aplicação do processo de Mineração de Textos. As hierarquias formadas, bem como os tópicos obtidos na execução deste processo são descritos na próxima seção.

\section{Análise das hierarquias de tópicos ob- tidas}

Após o processamento e validação, foi possível obter toda a hierarquia de tópicos extraída pela ferramenta Torch, processados em duas etapas, para documentos de eventos nacionais e, posteriormente, de eventos internacionais.

A Torch oferece recursos que permitem analisar os documentos associados aos tópicos, dispostos de forma hierárquica. Adicionalmente, a ferramenta também fornece uma medida de avaliação do documento (score), ou seja, o quão significativo é aquele documento em relação os tópicos selecionados. Essa medida, ilustrada na Figura 2, está relacionada à distância daquele documento ao centro do grupo a que ele foi atribuído.

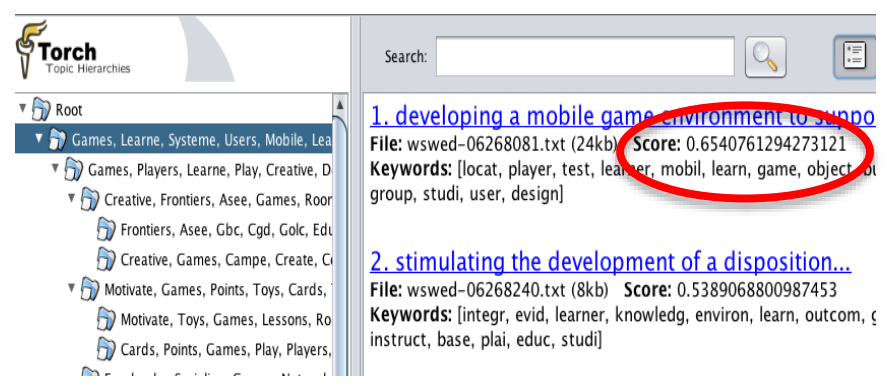

Figura 2: Score: medida de avaliação do documento em relação aos tópicos.

Desse modo, para simplificar a análise exploratória dos tópicos mais abordados, considerou-se inicialmente os tópicos com os maiores scores apresentados pela ferramenta Torch.

Para a análise exploratória, foram considerados os grupos com até no mínimo 20 documentos, ou seja os 9 maio- 
res grupos nacionais. Proporcionalmente foram analisados apenas os 6 maiores grupos internacionais pois devido à grande quantidade de documentos, a análise dos subgrupos mostrou-se adequada e relevante. Cada grupo, possui um conjunto de documentos e tópicos que descrevem esses documentos. Na Tabela 2, estão os dados parciais que representam os grupos e subgrupos analisados e seus respectivos descritores (tópicos).

Vale ressaltar que os grupos selecionados possuem diferentes quantidades de documentos. Essa quantidade é significativa em relação à representatividade do descritor, ou seja, quanto menor a quantidade de documentos em um determinado grupo, mais específico são os descritores desse grupo. Em grupos maiores, a quantidade de temas abordados tende a ser maior e suas especializações estão dispostas nos subtópicos da hierarquia.

Tabela 2: Grupos e subgrupos analisados e seus respectivos tópicos.

\begin{tabular}{|c|c|}
\hline \multicolumn{2}{|c|}{ Tópicos (descritores) } \\
\hline Internacionais & Nacionais \\
\hline $\begin{array}{l}\text { Grupo 1: } 633 \text { documentos } \\
\text { Grupo 1.1: game, gameful, } \\
\text { gamification, play, mobile, } \\
\text { desian competitive }\end{array}$ & $\begin{array}{l}\text { Grupo 1: } 102 \text { documentos } \\
\text { curso, disciplina, projeto, } \\
\text { estudo, software, computação, } \\
\text { DOJO, problema }\end{array}$ \\
\hline $\begin{array}{l}\text { Grupo 1.2: adaptable, } \\
\text { contexts, system, content, } \\
\text { users, objective, designer, } \\
\text { modeler, informative }\end{array}$ & $\begin{array}{l}\text { Grupo 2: } 86 \text { documentos } \\
\text { jogo, software, desafio, educa- } \\
\text { ção, fase, ambiente, estimulo, } \\
\text { ponto, fase }\end{array}$ \\
\hline $\begin{array}{l}\text { Grupo 1.3:, visualize, } \\
\text { interactive, simulate, tools, } \\
\text { collaborative }\end{array}$ & $\begin{array}{l}\text { Grupo 3: } 66 \text { documentos } \\
\text { social, rede, digital, tecnologia, } \\
\text { espaço, facebook }\end{array}$ \\
\hline $\begin{array}{l}\text { Grupo 2: } 398 \text { documentos } \\
\text { Grupo 2.1: challenges, } \\
\text { software, testing, develop- } \\
\text { er, code, software }\end{array}$ & $\begin{array}{l}\text { Grupo 4: } 38 \text { documentos } \\
\text { agente, lógica, sistema, ambi- } \\
\text { ente, aprendizado, ontologia, } \\
\text { semântica, multiagente, meta- } \\
\text { dado }\end{array}$ \\
\hline $\begin{array}{l}\text { Grupo 2.2: teams, project, } \\
\text { software, teamworks, } \\
\text { agile, group }\end{array}$ & $\begin{array}{l}\text { Grupo 5: } 33 \text { documentos } \\
\text { texto, fórum, analisado, ferra- } \\
\text { menta, documento, resultado, } \\
\text { colaborativa, leitura, minera- } \\
\text { ção }\end{array}$ \\
\hline $\begin{array}{l}\text { Grupo 3: } 391 \text { documentos } \\
\text { problems, programs, solve, } \\
\text { coders, students, grades }\end{array}$ & $\begin{array}{l}\text { Grupo 6: } 27 \text { documentos } \\
\text { formação, fluência, TIC, profis- } \\
\text { sional, docente, educação, } \\
\text { tecnologia, informação }\end{array}$ \\
\hline $\begin{array}{l}\text { Grupo 4: } 247 \text { documentos } \\
\text { researching, programs, } \\
\text { women, graduate, years, } \\
\text { student, female }\end{array}$ & $\begin{array}{l}\text { Grupo 7: } 25 \text { documentos } \\
\text { objeto, aprendizado, conteúdo, } \\
\text { conceito }\end{array}$ \\
\hline $\begin{array}{l}\text { Grupo 5: } 202 \text { documentos } \\
\text { robotic, computer, pro- } \\
\text { gram, computer, pro- } \\
\text { grams, lego }\end{array}$ & $\begin{array}{l}\text { Grupo 8: } 25 \text { documentos } \\
\text { Simulado, memória, servidor, } \\
\text { ferramenta }\end{array}$ \\
\hline
\end{tabular}

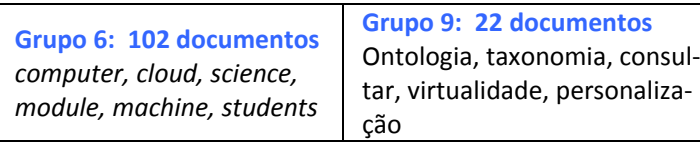

Além disso, grupos com poucas quantidades de documentos podem indicar comunidades de pesquisa. De modo geral, as comunidades são formadas por pesquisadores que investigam um mesmo assunto sob diferentes perspectivas, que eventualmente são de uma mesma região do país ou até de um mesmo laboratório de pesquisa.

Nas próximas seções são abordados detalhadamente os assuntos relacionados aos maiores grupos extraídos da hierarquia de tópicos, tanto no contexto nacional como internacional.

\subsection{Eventos e periódicos internacionais}

Os eventos internacionais foram agrupados em 17 grupos dispostos em um modelo hierárquico de até 8 níveis de profundidade. Dos 29 grupos, o maior deles conta com 633 e o menor com 5 documentos. O principais grupos analisados possuem respectivamente $633,398,391,247,202,102$ documentos em cada grupo. Na Figura 4 são ilustrados todos os grupos gerados.

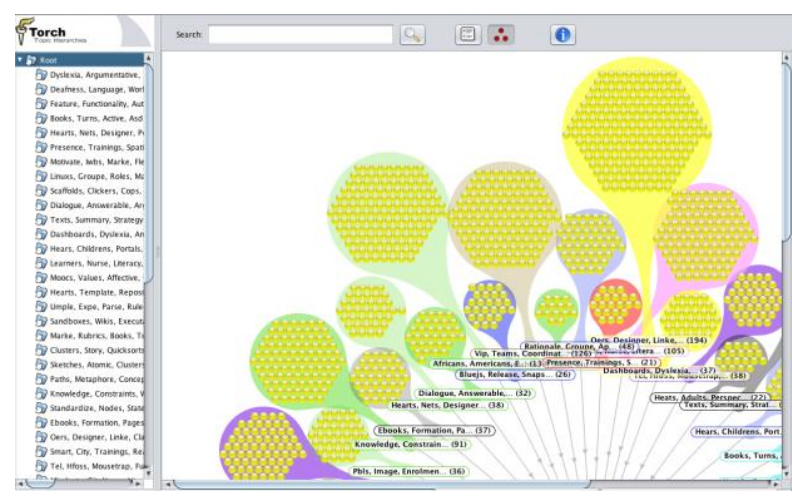

Figura 4: Hierarquia de tópicos dos eventos internacionais.

Considerando os agrupamentos extraídos, observa-se que os assuntos mais abordados pela comunidade no contexto internacional são:

1 Jogos educacionais, dispositivos móveis (mLearning), sistemas adaptativos e conteúdo educacional, simuladores:

- Jogos educacionais na web ou em dispositivos móveis. Utilização de tutores inteligentes ou agentes para modelar o comportamento de jogadores. Pesquisas que investigam acessibilidade em jogos educacionais. Jogos associados a análises em tempo real (learning analytics). Experimentos que avaliam a efetividade da aprendizagem baseada em jogos (game based learning). 
- Definição de uma plataforma de aprendizagem baseada em problema para dispositivos móveis (problem based learning). Investigação sobre os principais desafios e riscos da utilização de dispositivos móveis durante o ensino e aprendizado. Inclusão da disciplina de computação móvel na grade curricular de ciências da computação.

- Adaptação e personalização de conteúdos educacionais para sua utilização dispositivos móveis. Utilização de mapas conceituais para o compartilhamento colaborativo do conhecimento. Reutilização de conteúdos educacionais para ambientes móveis utilizando SCORM.

- Mapas conceituais para a construção do conhecimento. Ontologias utilizada para auxiliar a estruturação do domínio e para auxiliar agentes pedagógicos.

- Pesquisas sobre a criação e utilização de laboratórios virtuais para o aprendizado de matemática, física, entre outros. Definição de simuladores para diversas disciplinas como eletrônica, microeletrônica, termoeletrônica, compiladores, entre outras.

É importante observar que este é o maior grupo da hierarquia e, por isso, existem assuntos de diferentes contextos. Esses assuntos referem-se aos subgrupo da hierarquia principal.

\section{Desafios e práticas, trabalho em grupo:}

- Estudos de casos relatando as experiências e desafios no ensino de engenharia de software, teste de software e gerenciamento de projetos, entre outras disciplinas.

- Estudo que investigam formas de desenvolver habilidades para trabalhar em grupos, principalmente em contextos de desenvolvimento de software. Utilização de metodologias ágeis para trabalhos em grupo.

\section{Resolução de problemas:}

- Experiências e estudos de casos comparando problemas do mundo real versus online.

- Estudos que demonstram a eficiência de técnicas como desenvolvimento ágil e DOJO como forma de promover melhorias no aprendizado de desenvolvimento de software.

- Estudos de casos avaliando melhorias no ensino e aprendizado quando há revisão em pares (peer review).

- Demonstração de ferramentas em contextos específicos.

- Definição de técnicas, programas e scripts para melhorar questões funcionais de ambientes educa- cionais.

\section{Ensino, abordagens e metodologias educacionais:}

- Pesquisas com o foco em novas experiências, desafios e tendências. A grande maioria descreve estudos de casos e experiências em sala de aula, seja com mudanças na forma de ensinar, alterações em atividades curriculares ou até mesmo utilização de novas práticas, técnicas e tecnologias.

- Pesquisas que abordam análises exploratórias de abordagens e métodos com foco em programas de graduação e pós-graduação, análise da efetividade do ensino em contextos diferenciados.

- Pesquisas com o objetivo de incorporar boas práticas ao currículo de disciplinas. Várias abordagens são consideradas, principalmente relacionadas às engenharias.

- Pesquisas que evidenciam tendências e perspectivas. Um exemplo de tendência abordado são os trabalhos que enfatizam formas de melhorar a adesão a cursos de engenharia por grupos do sexo feminino ou, ainda, descrição de preditores para reter estudantes de engenharia do sexo feminino.

\section{Robótica, legos e programação:}

- Utilização de lego como apoio ao ensino e aprendizado. Nesse contexto os legos podem ser utilizados como forma de motivação e retenção de alunos.

- Criação de robôs sociais de baixo custo para auxiliar disciplinas como filosofia e psicologia.

- Estudos de casos de uso de robótica em cursos do ensino fundamental e cursos de graduação como engenharia elétrica, engenharia mecatrônica, curso básico de programação, entre outros.

\section{Computação, programação:}

- Melhorias no ensino e aprendizado de diferentes linguagens de programação.

- Inclusão da disciplinas como computação na nuvem, computação paralela, desenvolvimento para dispositivos móveis para cursos de ciências da computação e engenharia da computação.

- Análise de frameworks e ambiente de programação considerando o contexto pedagógico.

- Uso de programas computacionais como apoio ao ensino e aprendizado.

\subsection{Eventos e periódicos nacionais}

Os eventos nacionais possuem 74 pequenos grupos, conforme ilustrado na Figura 5. Dentre estes grupos, o maior deles possui 102 documentos e o menor possui 3 
documentos. Cada um dos grupos ainda possuem alguns subgrupos dispostos em um modelo hierárquico de até 5 níveis de profundidade.

Dentre os assuntos mais abordados pela comunidade no contexto nacional, seguindo a ordem decrescente de importância, destacam-se:

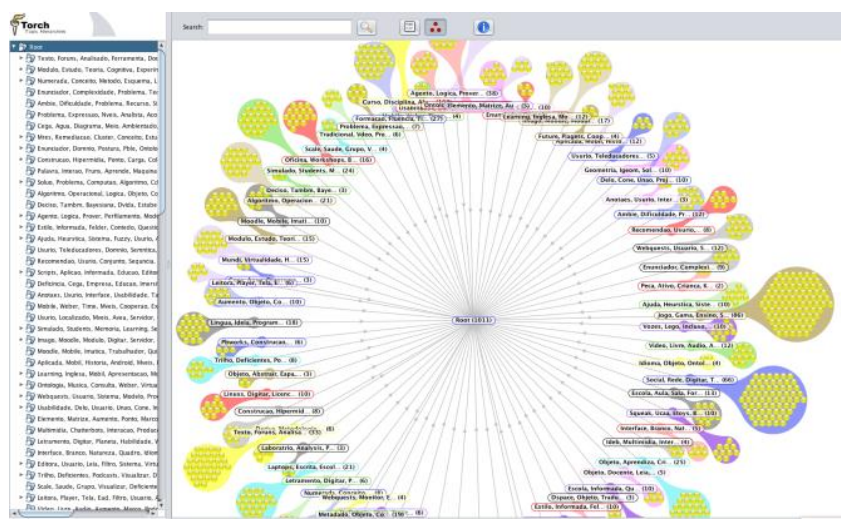

Figura 5: Hierarquia de tópicos dos eventos nacionais.

1 Utilização de software como auxílio ao ensino e aprendizado e avaliações de sua efetividade:

- Desenvolvimento de ferramentas, algumas delas como software livre, como forma de apoio ao processo educacional.

- Ambientes educacionais e ferramentas de apoio.

- Estudos de caso que relatam experiências no ensino de diversas disciplinas tanto no ensino médio como no ensino superior. Por exemplo, matemática, engenharia de software, teste de software, informática, programação, entre outras.

- Utilização de diferentes técnicas e metodologias para apoiar o ensino. Por exemplo, Scrum, PBL e DOJO.

- Avaliação de cursos à distância considerando tutores/professores e ambientes educacionais.

De modo geral, as pesquisas evidenciadas neste grupo demonstram resultados obtidos considerando diferentes problemas relatados ao ensinar disciplinas, principalmente em ciências exatas.

\section{Jogos educacionais:}

- Jogos educacionais aplicados em diferentes estratégias pedagógicas e contextos de aprendizagem.

- Jogos disponibilizados em ambientes virtuais de aprendizagem para motivar e envolver o aluno no assunto de interesse.
- Jogos para apoiar disciplinas como física, matemática, química, biologia, entre outras.

\section{Comunidades e redes sociais:}

- Estudos exploratórios e pesquisas que relatam estudo de casos utilizando redes sociais.

- Desenvolvimento ou análise de plataformas colaborativas como apoio ao ensino e aprendizado.

- Utilização de funcionalidades do facebook como ferramenta social para complementar o ensino e aprendizado.

\section{Agentes inteligentes:}

- Agentes inteligentes utilizados em análises e validações de ambientes educacionais.

- Sistemas multi-agentes para apoiar a recomendação de conteúdo e a personalização do ambiente educacional.

- Ambientes educacionais com comunicação feita a por meio de agentes inteligentes. Ambientes educacionais com agentes pedagógicos.

- Pesquisas que utilizam agentes inteligentes, metadados e ontologias simultaneamente para catalogação de objetos de aprendizagem.

\section{Mineração de textos/dados educacionais e cola- boração:}

- Utilização de mineração de textos para apoiar a escrita colaborativa, ajudar o aluno na prática da leitura, letramento, autoria, entre outros. A mineração de texto também é utilizada na análise de dados textuais em wikis e fóruns de discussão.

- Utilização de algoritmos de comparação textual para descobrir similaridades e evitar o plágio em atividades educacionais.

- Desenvolvimento ou análise de plataformas colaborativas como apoio ao ensino e aprendizado. Ambientes educacionais como apoio à colaboração.

\section{Formação:}

- Formação continuada de professores para uso pedagógico de tecnologias digitais.

- Utilização de tecnologia da informação e comunicação nas práticas docentes aplicadas em diversas disciplinas.

- Análises de perfis profissionais adequados à utilização de tecnologias como apoio ao ensino e aprendizado.

- Utilização de técnicas e tecnologias para auxiliar e a formação de professores por meio de ambientes virtuais de aprendizagem. 


\section{Objetos de aprendizagem:}

- Temas relacionados à criação, utilização, empacotamento (SCORM) e armazenamento em repositórios desses objetos.

- Objetos de aprendizado para apoiar o ensino de diversas disciplinas como matemática, português, entre outras.

- Definições de repositórios de objetos de aprendizado.

\section{Simuladores:}

- Ambientes de simulação para disciplinas como sistemas operacionais, estrutura de dados, arquitetura de computadores, entre outras.

\section{Ontologias e mapas conceituais:}

- Ontologias para modelar domínio de conhecimento. Utilizada também para recuperação de informação e/ou realizar inferências como apoio à recomendação.

- Pesquisas que buscam realizar melhorias na localização, recuperação, catalogação e reutilização de objetos de aprendizagem utilizando ontologias.

- Ontologia como representação do conhecimento ou personalização de conteúdo educacional.

É importante destacar que grande parte dos assuntos abordados possuem pesquisas que descrevem estudos de caso. De modo geral, tais estudos buscam relatar experiências de alunos seja na adoção ou avaliação de ambientes e sistemas de apoio ao ensino e aprendizado, ou até mesmo abrangendo discussões relacionadas à atualização do modelo de ensino para incluir disciplinas mais adequadas ao contexto atual da informática/computação.

Além disso, também é importante considerar tópicos que aparecem em um grupo, mas não em outro. Isso significa apenas que no primeiro grupo (que contém o tópico) a pesquisa é emergente, pois o tópico descreve uma quantidade de documentos.

É importante destacar que o objetivo desta pesquisa foi realizar uma análise exploratória e não uma comparação direta. Sob essa perspectiva, a análise exploratória dos tópicos abordados em pesquisas internacionais e nacionais representa um forte indicativo do que está sendo investigado na área de Informática na Educação. Desse modo, é possível traçar um panorama de quais assuntos estão sendo mais investigados, além de contribuir para o melhor entendimento da área.

\section{Conclusões e Trabalhos Futuros}

Neste artigo, foi realizada uma análise exploratória dos tópicos de pesquisa emergentes na área de Informática na Educação.

Como apoio a essa análise, foram construídas hierarquias de tópicos a partir de publicações em conferências nacionais e internacionais na área realizadas nos últimos anos. A partir dessas hierarquias, foram analisados os grupos hierárquicos formados, detectando aspectos importantes sobre a pesquisa realizada na área.

Considerando as análises efetuadas, é possível observar que a área de Informática na Educação possui forte vertente de pesquisas direcionadas por estudos de caso ou provas de conceito. Como consequência, tem-se o desenvolvimento de melhorias no processo de ensino e aprendizagem, tanto no contexto nacional como internacional.

Além disso, é importante destacar que a comunidade científica vêm discutindo cada vez mais a inclusão e a atualização das disciplinas para considerar a informática como apoio ao processo educacional.

Um dos assuntos bastante evidenciado, em ambos os cenários, refere-se aos jogos educacionais. As pesquisas nesse tema estão diretamente associadas a diversas áreas de atuação. Dentre as áreas de pesquisa, destacam-se jogos tanto na web como em dispositivos móveis, com análises em tempo real (learning analytics), entre outros.

Além disso, jogos educacionais são utilizados e analisados sob diferentes estratégias pedagógicas. De modo geral, as pesquisas associadas a esse tópico buscam sempre motivar e envolver o aluno no assunto de interesse a fim de proporcionar uma aprendizagem mais efetiva.

Pesquisas destinadas ao projeto e desenvolvimento de ambientes educacionais e ferramentas inteligentes e adaptáveis também são emergentes em ambos os cenários. Além disso, a utilização de dispositivos móveis é enfatizada nesse contexto com o objetivo de personalização e adaptação. Sistemas tutores inteligentes, agentes e ontologias são importantes tópicos de interesse, tanto no Brasil como no exterior.

Outro assunto também emergente em ambos os cenários refere-se à utilização técnicas e metodologias como DOJO e metodologias ágeis para apoiar o ensino e aprendizado, principalmente em contextos colaborativos.

Por fim, com menor enfoque no cenário nacional estão as pesquisas que utilizam robótica como apoio ao ensino e aprendizado. 
Como trabalhos futuros, pretende-se expandir a pesquisa efetuada para descobrir subgrupos de pesquisas que se especializam em determinados assuntos. Uma outra vertente dessa pesquisa pode considerar, ainda, a utilização de referências em documentos como fonte de informações para possibilitar a análise da influência de grupos de pesquisa a partir das referências presentes nos documentos.

\section{Referências}

[1] A. K. Jain e R. C. Dubes. Algorithms for clustering data. Prentice-Hall, Inc., Upper Saddle River, NJ, USA, 1988.

[2] B. M. Nogueira, M. F. Moura, M. S. Conrado, R. G. Rossi, R. M. Marcacini, e S. O. Rezende. Winning some of the document preprocessing challenges in a text mining process. In WAAMD '08: Anais do IV Workshop em Algoritmos e Aplicações de Mineração de Dados - SBBD '08: XXIII Simpósio Brasileiro de Banco de Dados, pages 10-18. Porto Alegre: SBC, 2008.

[3] Capes. Qualis periódicos. 2012.

[4] C. D. Manning e H. Schtze. Foundations of Statistical Natural Language Processing. MIT Press, Cambridge, MA, EUA, 1999.

[5] C. G. von Wangenheim, D. Kochanski, and R. Savi. Revisão sistemática sobre avaliação de jogos voltados para aprendizagem de engenharia de software no Brasil. FEES: Fórum de Educação em Engenharia de Software, 2009.

[6] C. Romero e S. Ventura. Educational data mining: a review of the state of the art. Systems, Man, and Cybernetics, Part C: Applications and Reviews, IEEE Transactions on, 2010.

[7] D. Lawrie e W. B. Croft. Discovering and comparing topic hierarchies. In RIAO '00: Proceedings of the 6th Recherche d'Informations Assistee par Ordinateur, pages 314-330, 2000.

[8] E. T. Matsubara, C. A. Martins, e M. C. Monard. Pre-text: uma ferramenta para preprocessamento de textos utilizando a abordagem bag-of-words. Technical Report 209, Instituto de Ciências Matematicas e de Computação - USP São Carlos, 2003.

[9] G. Salton e C. Buckley. Term weighting approaches in automatic text retrieval. Technical report, Ithaca, NY, EUA, 1987.

[10] H. P. Luhn. The automatic creation of literature abstracts. IBM Journal os Research and Devel- opment, 2(2):159-165, 1958.

[11] I. Witten, E. Frank, e M. Hall. Data Mining: Practical machine learning tools and techniques. Morgan Kaufmann, 2011.

[12] J. L. Neto, A. D. Santos, C. A. A. Kaestner, e A. A. Freitas. Document clustering and text summarization. In L. T. P. A. Company, editor, Proceedings of the 4th International Conference on Practical Applications of Knowledge Discovery and Data Mining - PADD2000, pages 41-55, 2000.

[13] J. B. MacQueen. Some methods for classication and analysis of multivariate observations. In L. M. L. Cam and J. Neyman, editors, Proceedings of the V Berkeley Symposium on Mathematical Statistics and Probability, volume 1, pages 281297. University of California Press, 1967.

[14] Miiller, A. e Dorre, J. (1999). The taxgen framework: Automating the generation of a taxonomy for a large document collection. Em HICSS '99: Proceedings of the Thirty-Second Annual Hawaii International Conference on System Sciences-Volume 2, pagina 2034, Washington, DC, USA. IEEE Computer Society.

[15] R. M. Marcacini e S. O. Rezende. Incremental Construction of Topic Hierarchies using Hierarchical Term Clustering. In SEKE'2010: Proceedings of the 22nd International Conference on Software Engineering and Knowledge Engineering, Redwood City, San Francisco, USA. KSI Knowledge Systems Institute, pages 553-558, 2010.

[16] M. F. Porter. An algorithm for sux stripping. Readings in Information Retrieval, pages 313316, 1997.

[17] N. F. F Ebecken, M. C. S. Lopes e M. C. A. Costa. Mineração de Textos. In S. O. Rezende, editor, Sistemas Inteligentes: Fundamentos e Aplicações, capítulo 13, páginas 337-370. Manole, 1 edição, 2003.

[18] R. Feldman e J. Sanger. The text mining handbook: advanced approaches in analyzing unstructured data. Cambridge University Press, 2006.

[19] R. Marcacini e S. Rezende. Torch: a tool for building topic hierarchies from growing text collection. In WTA-2010: IX Workshop on Tools and Applications. In 8th Brazilian Symposium on Multimedia and the Web (Webmedia), pages 133-135, 2010. 
[20] R. Gil-Garcia and A. Pons-Porrata. Dynamic hierarchical algorithms for document clustering. Pattern Recognition Letters, 31(6):469 - 477, 2010.

[21] Romero, Cristóbal, and Sebastián Ventura. "Educational data mining: a review of the state of the art." Systems, Man, and Cybernetics, Part C: Applications and Reviews, IEEE Transactions on 40.6 (2010): 601-618.

[22] S. O. Rezende, J. B. Pugliesi, E. A. Melanda, e M. F. Paula. Mineração de dados. In S. O. Rezende, editor, Sistemas Inteligentes: Fundamentos e Aplicações, chapter 12, pages 307-335. Manole, 1 edition, 2003.

[23] Murray, Tom. "An Overview of Intelligent Tutoring System Authoring Tools: Updated analysis of the state of the art." Authoring tools for advanced technology learning environments. Springer Netherlands, 2003. 491-544.

[24] Tang, L., Liu, H., Zhang, J., Agarwal, N., e Salerno, J. J. (2008). Topic taxonomy adaptation for group proling. ACM Transactions on Knowlowledge Discovery from Data, 1(4):1-28.

[25] T. K. Shih. Distance education technologies: current trends and software systems. In Cyber Worlds, 2002. Proceedings. First International Symposium on, pages 38-43. IEEE, 2002.

[26] U. M. Fayyad, G. Piatetsky-Shapiro, e P. Smyth. Knowledge discovery and data mining: Towards a unifying framework. In $K D D$ '96: Proceedings of II International Conference on Knowledge Discovery and Data Mining, pages 82-88, EUA, 1996. AAAI Press.

[27] Y. Zhao e G. Karypis. Evaluation of hierarchical clustering algorithms for document datasets. In CIKM '02: Proceedings of the 11th International Conference on Information and Knowledge Management, pages 515-524, New York, NY, USA, 2002. ACM.

[28] Zavitsanos, E., G. Paliouras, e G. A. Vouros (2011). Non-parametric estimation of topic hierarchies from texts with hierarchical dirichlet processes. Journal of Machine Learning Research 12, 2749-2775. 